\title{
Why the first laser worked as designed (and is still kicking today)
}

\begin{abstract}
Andrew H. Rawicz rawicz@sfu.ca

School of Engineering Science, Simon Fraser University, BC, Canada

Theodore Maiman, the inventor of the laser, was both a physicist and an engineer. One can speculate that this combination of backgrounds was the main reason for the successful design, construction, and demonstration of the ruby laser in May 1960. The reasons for this success - as stated by Maiman in discussions with the present author - include some basic rules of elegant engineering design: understand what you want to make, understand the physics behind it, understand the nature of the materials to be used for fabrication, and finally, be a minimalist - simplify. Even now, the elegance and simplicity of the design of the first laser is evident upon viewing. The following text will try to gather and clarify all the components necessary for an invention such as the laser, classified by Nature magazine as one of twenty one most important inventions of twentieth century. [DOI: 10.2971/jeos.2010.10046s]
\end{abstract}

Keywords: laser invention, Theodore Maiman

\section{THEODORE MAIMAN AS A PERSON}

Full disclosure: the author of this article was a close friend of Theodore Maiman from 2001 until his death in May 5, 2007. Perhaps an informal psychological description of Maiman may be relevant to the explanation of this invention. For the sake of brevity only a few aspects of Maiman's (from now on referred to as "Ted") life will be brought up. Anyone interested in the full story should read "The Laser Odyssey" by Theodore Maiman, published by Laser Press in Blaine, Washington in 2000 [1]. Other valuable sources of of information on the laser and on the scientific and bibliographic history of the invention of the laser include [2]-[4].

During his professional life, which he spent in various divisions of AT\&T and Bell Telephone Laboratories, Ted's father Abe (Abraham) showed an inventive nature. A number of his inventions were applied in the car industry (early DC-toDC converter), medicine (electronic stethoscope), audio sound systems, and an invention of dial-by-radio system. To work more on his inventions and explore his creative ideas, he kept a small electronic shop in their home. This was where Ted was very early on introduced to the world of technology; a world which his father, participated in as an electrical engineer. Later, early in his teens, Ted started his first paying job in a small shop repairing appliances and radios. Repairing products requires a very thorough understanding of their functioning, a lesson which had a profound effect on Ted's life as a researcher, designer, discoverer, and inventor.

Because of his insatiable curiosity for an in-depth understanding of surrounding phenomena, Ted decided to broaden his formal education by studying both physics and electrical engineering at the University of Colorado at Boulder, where he graduated with a Bachelor of Science in Engineering Physics. After receiving a Masters of Science in Electrical Engineer- ing at Stanford, he was there accepted to the PhD program in Physics.

Working under the supervision of Willis Lamb, an acclaimed theoretical physicist, Ted learned the rigors of physical experimentation, redesigning and modifying the laboratory equipment used for his dissertation topic: "Microwave-Optical Investigations of the $3^{3} \mathrm{P}$ Fine Structure of Helium". He defended his thesis in 1955, the same year in which Willis Lamb was awarded his Nobel Prize. In his book Ted says: "The inspirational teachings of Professor Lamb were of inestimable value to my future career. I probably learned more from Willis Lamb than in all my prior course work combined. The knowledge that I gained from my thesis experiment was powerful in providing me with important tools that I needed later for developing the laser".

In 1956 Ted started working in the Hughes Atomic Physics department and quickly was selected to head the maser project. A maser is a device which amplifies microwaves and is somewhat similar to laser. The standard at that time, the solid-state maser, was a monstrosity weighing about $2500 \mathrm{~kg}$. Most of the weight was due to a huge electromagnet and a double Dewar cryo-cooling system with external liquid nitrogen and internal liquid helium layers. This huge infrastructure housed a microwave cavity, inside of which was a small maser crystal, a tiny synthetic ruby crystal. This crystal has the capacity of detecting and amplifying extremely weak microwave signals. Ted radically redesigned the maser and managed to reduce its weight by about 200 times to $12.5 \mathrm{~kg}$, increasing its' detecting efficiency at the same time. While working on the maser Ted learned enough to state: "Cryogenics is the killer, avoid it at all costs". The redesigned maser, although more practical than the elephant version, still required liquid nitrogen to operate properly. This need for cryo-cooling made the device vulner- 
able to competition and soon the ruby-maser was replaced by an electronic parametric amplifier. This exercise, nevertheless, prepared Ted even more to start seriously thinking about developing the laser. More psychological insight will be provided later in Section 3

\section{THE RACE TO THE LASER}

Although the thorough theoretical design of the conditions for lasing were provided by Vitalij Fabrikant in his doctoral dissertation (1940), he never constructed the laser. In December 1958 Townes and Schawlow published "Infrared and Optical Masers" in Physical Review [5]. They proposed potassium vapour as a lasing medium. The proposed material and laser construction based on their presented concept appeared to be completely unworkable (read: wrong), nevertheless the article stimulated a number of researchers to engage in the race to make a coherent light source. At that time, Ted was still making improvements to the maser. He joined the race in the second half of 1959 after managing to convince his Hughes bosses to allow him to work on the laser and provide him with a small grant $(\$ 50,000)$. Irnee $D^{\prime}$ Haenens, a masters student from the University of Southern California, was assigned to assist him. With those limited means and already being behind some of the powerful teams at Bell Labs, TRG, and MIT, Maiman had to think of a concept that was inexpensive and would not contain too many uncertainties. These considerations are listed and elaborated below.

\subsection{Solid state vs. gas}

Before the laser was made, nobody really knew what, if any, material would allow for a sustainable inverse population in atoms, although Purcel and Pound first reported the evidence of net stimulated emission in 1950. Most of the researchers engaged in the race towards the laser considered using gases or vapours. Use of the ruby was ruled out early, after a convincing statement by Schawlow during the Shawanaga Lodge Conference about it's unsuitability in Optical Maser (official name before the term "laser" was coined) action. In addition, Irwin Wieder from Westinghouse Research Laboratory measured quantum efficiency of ruby crystal and his result of $1 \%$ was published [6] in November 1959. The result was more than discouraging for anyone, including Ted. Fortunately Ted was familiar with synthetic rubies from his earlier work on masers and had an intuitive sense of their usefulness in a threelevel laser. After a short abandonment of ruby Ted re-measured its fluorescence with a new mono-chromator and the result was totally opposed to Wieder's 1\%; it was about $75 \%$. He decided to stick to ruby also for another reason, the "colour" or more correctly the wavelength which "pink" ruby fluoresced. It was red and thus easily observable. Ted knew from his earlier experiments that the ruby crystal was sturdy and resistant to mechanical stresses, and relatively easy to form and process. The decision was made. A number of other decisions about the mode of operation of the future laser were still to be made.

\subsection{Pulse mode vs. continuous}

Most of the groups engaged in the race towards the laser (optical maser) considered working on CW lasers. Ted wanted to use a photo-flash lamp as a pumping source and this choice determined pulse mode. From his and other researchers' earlier work on ruby's optical properties, Ted knew that the wavelength of the fluorescence was temperature dependent. Continuous pumping would affect crystal temperature and, even worse, its uniformity, thus creating stresses. A temperature stabilizing addition would be required. The decision was made - pulse mode. CW mode ruby laser was developed 5 years later by Ted's collaborator in Hughes, Viktor Evtuhov.

\subsection{Short lasing medium (stubby ruby)}

For almost every physicist involved in designing the laser, it was obvious; the longer the lasing medium, the better. The optical gain in fluorescing material is a function of the pathlength along the cavity (Fabry-Perot resonator):

$$
G=T_{\text {surf }} \exp \left[C_{\text {mat }} d\right],
$$

where $T_{\text {surf }}$ is surface transmission factor, $C_{\text {mat }}$ is a material and pumping source dependent constant, and $d$ is the optical path-length.

In the late 1950s, ruby crystals could reliably be fabricated of even $1 \mathrm{~m}$ lengths, although not the best quality yet. Ted intuitively realized that the precision of fabricating ideally parallel end-mirrors would pose a difficulty and for a long rod this unparallelism might destroy any length-related gain due to the escape of deflected photons. He thus chose a rod only $2 \mathrm{~cm}$ long, with about $1 \mathrm{~cm}$ in diameter [7].

\subsection{Non-cryogenic}

Optical properties of lasing media vary with temperature changes. The theoretical calculations of inverse population in most media considered for lasers at the time of the "race" indicated the necessity of using very low temperatures (cryocooling). Schawlow insisted that the ruby also required cryogenics to function. The mistake in his thinking was that only dark ruby with its "four level system" was an eventual candidate. Ted recalculated the conditions for the pink ruby and was confident that room temperature was sufficient, particularly if a pulse mode was applied. He avoided the "killer" cryogenics.

\section{3 "GOING AGAINST THE GRAIN"}

Today, for physicists and engineers working with lasers the four conditions presented above seem obvious, almost trivial. Before the laser was invented these conditions were all under question. At the symposium "Laser Celebration - Tribute to Theodore Maiman" held on May 15 and 16, 2010 in Vancouver, Canada [8], one of the speakers, Dan Gelbart (founder of laser based industrial printing machines manufacturing company CREO Products and a prolific inventor), examined how the psychological features of Ted enabled him to make the invention of the laser before others did. Ted had the courage 


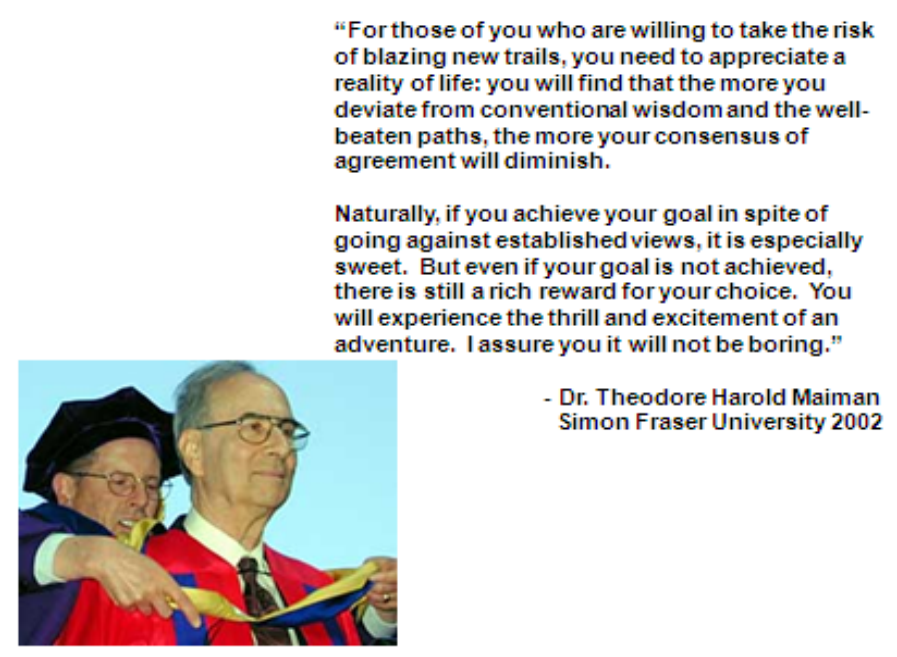

FIC. 1 Extract from Theodore Maiman's convocation speech and accompanying photograph.

to rely on his own assessment of approaches to solve problems. He did not bend to the pressure of high-profile skeptics, who from the height of their positions in the science world expressed negative opinions about his choice of materials, geometry of the design, modes of operations, etc., as described in Sections 2.1 to 2.4. He was not free of doubts and even after the first demonstration of the laser and the collection of its' measurement data, Ted asked Amnon Yariv, then on sabbatical from Bell Labs, for his interpretation. He felt relieved when Amnon stated after going through the results, "Maiman, it looks like you have a laser" [9]. When making a convocation speech after receiving an Honorary Doctorate from Simon Fraser University in 2002, Ted encouraged students to think on their own. Figure 1 shows a short excerpt from his convocation speech:

Some fellow professors did not like this encouragement of students to rebel against authorities, but in the author's opinion this short quote is a good description of Ted as a character. Figure 2 shows a photography of Ted and Irnee D'Haenens, showing the First Laser 25 years after its invention.

The First Laser was brought to this Vancouver Symposium (see Figure 3) by Kathleen (Ted's wife) and successfully fired several times on a projection screen. But what the heck, it is only 50 years old.

\section{References}

[1] T. Maiman, The Laser Odyssey, Theodore Maiman - Creator of the World First Laser (Laser Press, Blaine, WA, 2000).

[2] M. Bertolotti, The History of the Laser (Institute of Physics Publishing, Bristol and Philadelphia, 2005).

[3] J. Hecht, Beam: The Race to Make the Laser (Oxford University Press, 2005).

[4] A. H. Rawicz, "Theodore Harold Maiman and the invention of laser"

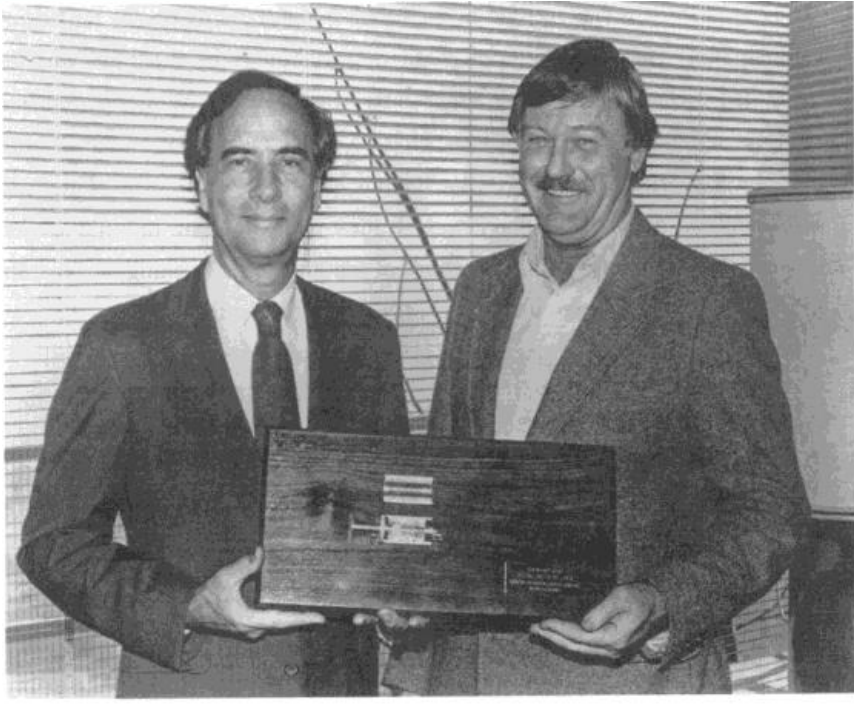

FIG. 2 Ted and Irnee D'Haenens proudly showing the First Laser 25 years later.

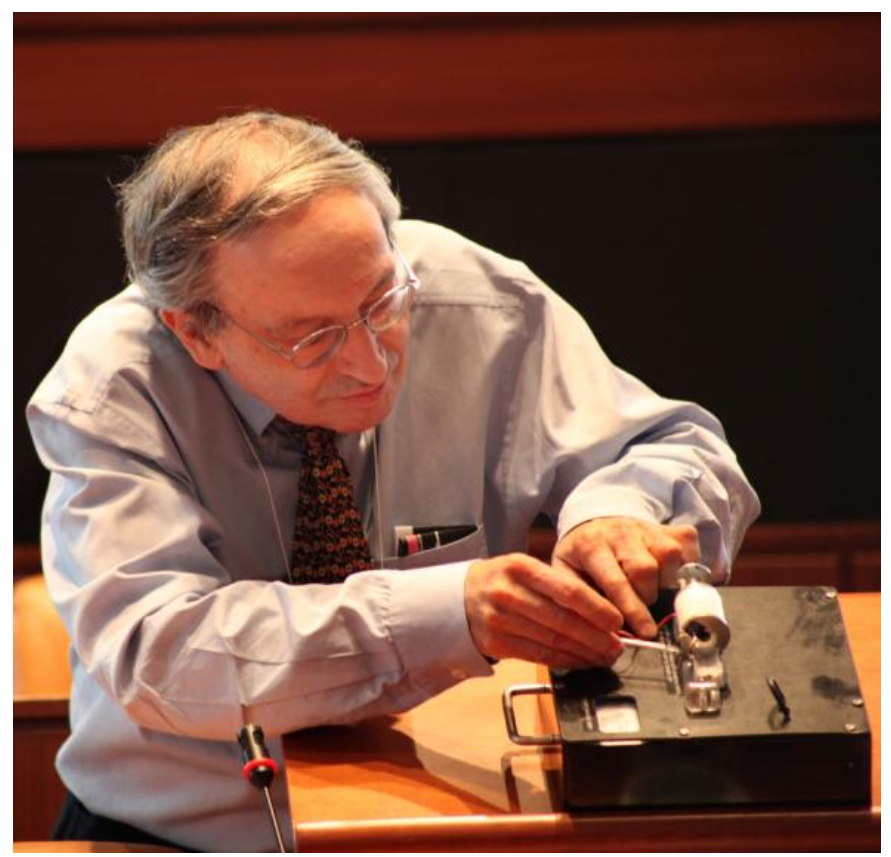

FIG. 3 Dan Gelbart preparing the First Laser for firing during the Vancouver Symposium (Photography courtesy of Professor Glenn Chapman, SFU).

Proc. SPIE 7138, 1-7 (2008).

[5] A. L. Schawlow, and C. H. Townes, "Infrared and Optical Masers" Phys. Rev. 112, 1940-1949 (1958).

[6] I. Wieder, "Solid State, High Intensity Monochromatic Light Sources" Rev. Sci. Instrum. 30, 995-996 (1959).

[7] Personal communication with T. Maiman, Vancouver, (2003).

[8] Laser Celebration - Tribute to Theodore Maiman symposium, Simon Fraser University, Vancouver, British Columbia, May 15 and 16, 2010 http://fas.sfu.ca/MaimanLaserSymposium/.

[9] Personal communication with A. Yariv, Prague, (2008). 